\title{
MULTIFRACTAL ANALYSIS OF THE BRJUNO FUNCTION
}

\author{
STÉPHANE JAFFARD AND BRUNO MARTIN
}

\begin{abstract}
The Brjuno function $B$ is a 1-periodic, nowhere locally bounded function, introduced by J.-C. Yoccoz because it encapsulates a key information concerning analytic small divisor problems in dimension 1. We show that $T_{\alpha}^{p}$ regularity, introduced by Calderón and Zygmund, is the only one which is relevant in order to unfold the pointwise regularity properties of $B$; we determine its $T_{\alpha}^{p}$ regularity at every point and show that it is directly related to the irrationality exponent $\tau(x)$ : its $p$-exponent at $x$ is exactly $1 / \tau(x)$. This new example of multifractal function puts into light a new link between dynamical systems and fractal geometry. Finally we also determine the Hölder exponent of a primitive of $B$.
\end{abstract}

Keywords: Brjuno function, continued fractions, irrationality exponent, Diophantine approximation, $T_{\alpha}^{p}$ regularity, $p$-exponent, multifractal analysis.

AMS classification: 11A55, 11J70, 11K50, 26A15, 26A30, 28A80, 37F50.

Acknowledgements: The authors thank Yves Meyer and the anonymous referee for many remarks on previous versions of this text.

Stéphane Jaffard is supported by ANR Grant MULTIFRACS ANR-16CE33-0020 and Bruno Martin by ANR Grant MUDERA ANR-14-CE34-0009.

\section{INTRODUCTION}

Let $x$ be an irrational number in $(0,1)$, and let

$$
x=\left[0 ; a_{1}, \ldots, a_{n}, \ldots\right]
$$

denote its continued fraction expansion. The convergents $p_{n} / q_{n}$ of $x$ are

$$
\frac{p_{n}}{q_{n}}=\frac{1}{a_{1}+\frac{1}{a_{2}+\frac{1}{\ddots \cdot+\frac{1}{a_{n}}}}}
$$

Addresses: Stéphane Jaffard: Université Paris Est, Laboratoire d'Analyse et de Mathématiques Appliquées, CNRS UMR 8050, UPEC, Créteil, France, jaffard@u-pec.fr Bruno Martin: Université du Littoral Côte d'Opale, EA 2797, Laboratoire de Mathématiques Pures et Appliquées Joseph Liouville, F-62228 Calais, France, Bruno.Martin@univ-littoral.fr . 
(following the standard tradition, we will not write explicitly the dependency of $p_{n}, q_{n}$ and $a_{n}$ in $x$ except when it will be needed). The Brjuno function at $x$ is

$$
B(x)=\sum_{n=0}^{\infty}\left|p_{n-1}-q_{n-1} x\right| \log \left(\frac{p_{n-1}-x q_{n-1}}{q_{n} x-p_{n}}\right),
$$

where, by convention,

$$
\left(p_{-1}, q_{-1}\right)=(1,0), \quad\left(p_{0}, q_{0}\right)=(0,1), \quad \text { and } \quad\left(p_{1}, q_{1}\right)=\left(1, a_{1}\right)
$$

so that the first term in (2) is $\log (1 / x)$. The Brjuno function is extended by periodicity on $\mathbb{R}-\mathbb{Q}$.

The Brjuno function plays an important role in the theory of holomorphic dynamical systems: It was first introduced by J.-C. Yoccoz, see [35], because of the information that it yields concerning analytic small divisor problems in dimension 1: Following C. L. Siegel [34], A. D. Brjuno [8] and J.-C. Yoccoz [35. germs with linear part $e^{2 i \pi x}$ are analytically conjugate to a rotation if and only if $x$ is a Brjuno number, i.e. if $x \notin \mathbb{Q}$ and if the series defining $B(x)$ is convergent.

B. Marmi, P. Moussa and J.-C. Yoccoz determined the optimal global regularity of $B$, showing that it belongs to BMO, see [23]. The Marmi-MoussaYoссоz conjecture is another regularity problem related with the Brjuno function: It states that the sum of $B$ and the logarithm of the conformal radius of the Siegel disk of a monic quadratic polynomial is $C^{1 / 2}$, see [23] p. 267. Key steps towards its resolution have been obtained by X. Buff, D. Cheraghi and A. Chéritat, see [9, 14. Local properties of $B$ were recently investigated by M. Balazard and B. Martin in [3]: They showed that its Lebesgue points are precisely the Bruno numbers, and they obtained precise estimates of the average of $B$ over an interval, which will play a key-role in our study, see e.g (9).

We will complement these regularity results by performing the multifractal analysis of the Brjuno function. The multifractal analysis of a function $f$ usually consists into three steps:

- Choose a pointwise regularity exponent compatible with the global function space setting where $f$ is considered,

- determine the value taken by this exponent at every point,

- compute the Hausdorff dimensions $\mathcal{D}_{f}(H)$ of the sets of points where this exponent takes a given value $H$.

The function $H \rightarrow \mathcal{D}_{f}(H)$ is the multifractal spectrum of $f$. Multifractal analysis has also been developped in the setting of measures and even of distributions, see e.g. [5, 26, 29] and references therein.

Several clues indicate that the tools supplied by multifractal analysis are relevant for the Brjuno function: First it is a cocycle under the action of 
$P G L(2, \mathbb{Z})$, as a consequence of the remarkable functional equations

$$
\begin{aligned}
\forall x \in \mathbb{R} \backslash \mathbb{Q}, & B(x+1)=B(x), \\
\forall x \in(0,1) \backslash \mathbb{Q}, & B(x)=\log (1 / x)+x B(1 / x),
\end{aligned}
$$

see [24, 25]. This property is reminiscent of the behavior of the Jacobi theta function under modular transforms, which is the key ingredient in the determination of the pointwise exponent of the non-differentiable Riemann function $\mathcal{R}(x)=\sum \sin \left(\pi n^{2} x\right) / n^{2}$ [18], and of related trigonometric series [33]. Other trigonometric series also related to modular forms have been studied by I. Petrykiewicz in [30, 31]. Finally, (2) also indicates that Diophantine approximation properties should play a role in the local regularity properties of $B$. This was the case for $\mathcal{R}$ [18], and several of its generalizations investigated by F. Chamizo, I. Petrykiewicz, S. Ruiz-Cabello, and A. Ubis in [11, 12, 13], and by T. Rivoal and J. Roques in [32. Note that extremely few explicit deterministic functions playing an important role in mathematics have been proved to have a non-trivial multifractal spectrum: Most results in multifractal analysis are either of probabilistic or generic nature. Another motivation for performing such an analysis on $B$ is that, beyond the important role played by this function, our result establishes a new relationship between holomorphic dynamical systems on one side, and real analysis and geometric measure theory on the other.

In order to perform the multifractal analysis of $B$, a first question is to determine a pointwise exponent fitted to its study. As mentioned above, this will be a consequence of the choice of a right function space setting. The two notions of pointwise regularity most commonly used are the Hölder exponent, defined for locally bounded functions and the local dimension, defined for positive Radon measures (see Section 41). However, these exponents are not fitted to the analysis of the Brjuno function for the following reasons. First, $B$ is not locally bounded (i.e. does not coincide a.e. with a locally bounded function), because of the logarithmic singularities in (2) centered at all rational points (the series (2) is positive so that cancellations between terms cannot occur). As regards the local dimension, since $B$ is positive, we can interpret it as the density of a positive Radon measure, but its local dimension is constant so that it is not adapted to measure the variations of regularity that exist in $B$. On other hand, these variations will be put into light through the use of a third notion of pointwise regularity, introduced by Calderón and Zygmund see [10], which is fitted to the study of functions that belong to $L_{l o c}^{p}$.

Definition 1. Let $p \in[1,+\infty)$ and $\alpha \geq-1 / p$. Let $f \in L_{\text {loc }}^{p}(\mathbb{R})$, and $x_{0} \in \mathbb{R}$; $f$ belongs to $T_{\alpha}^{p}\left(x_{0}\right)$ if there exist $C>0$ and a polynomial $P$ of degree less than $\alpha$ (with $P \equiv 0$ if $\alpha<0$ ) such that, for $\rho$ small enough,

$$
\left(\frac{1}{2 \rho} \int_{x_{0}-\rho}^{x_{0}+\rho}\left|f(x)-P\left(x-x_{0}\right)\right|^{p} d x\right)^{1 / p} \leq C \rho^{\alpha} .
$$


The p-exponent of $f$ at $x_{0}$ is

$$
h_{f}^{p}\left(x_{0}\right)=\sup \left\{\alpha: f \in T_{\alpha}^{p}\left(x_{0}\right)\right\} .
$$

\section{Remarks:}

- The normalization chosen in (3) is such that the simple cusp singularities $\left|x-x_{0}\right|^{\alpha}$ have an Hölder and a $p$-exponent which take the same value $\alpha$ at $x_{0}$ (for any $p \geq 1$ ).

- Definition 1 is a natural substitute for pointwise Hölder regularity when functions in $L_{l o c}^{p}$ are considered. In particular, the $p$-exponent can take negative values down to $-d / p$, and typically allows to deal with singularities which are locally of the form $1 /\left|x-x_{0}\right|^{\gamma}$ for $\gamma<d / p$.

- The condition on the degree of $P$ (which is required to ensure uniqueness of $P$ ) implies that, if $\alpha \leq 0$, then $P=0$.

Let $p=1$; if $f \in L_{l o c}^{1}$, and if the left-hand side of (3) is a $o(1)$, then $x_{0}$ clearly is a Lebesgue point of $f$ and the constant term of $P$ is the Lebesgue value of $f$ at $x_{0}$, i.e. is

$$
\lim _{\rho \rightarrow 0} \frac{1}{2 \rho} \int_{x_{0}-\rho}^{x_{0}+\rho} f(x) d x .
$$

Indeed, if we denote by $D$ this constant term,

$$
\left|\frac{1}{2 \rho} \int_{x_{0}-\rho}^{x_{0}+\rho} f(x) d x-D\right| \leq \frac{1}{2 \rho} \int_{x_{0}-\rho}^{x_{0}+\rho}|f(x)-D| d x=o(1) .
$$

It follows that the 1-exponent measures the rate of convergence of the local averages (4) in the Lebesgue theorem. Therefore the determination of the 1exponent that we will perform can be interpreted as a quantitative sharpening of the theorem of M. Balazard and B. Martin stating that every Brjuno point is a Lebesgue point of the Brjuno function. This is our main motivation for focusing on the case $p=1$. However, in Section 4.2 we will deal with arbitrary $p$ s (and conclude that, at any point, the $p$-exponent is independent of $p$ ). The 1-exponent of $B$ at a point will be related with its (Diophantine) irrationality exponent.

Definition 2. Let $x_{0} \notin \mathbb{Q}$, and $p_{n} / q_{n}$ the sequence of convergents of the continued fraction expansion of $x_{0}$. Let $\tau_{n}\left(x_{0}\right)$ be defined by

$$
\left|x_{0}-\frac{p_{n}}{q_{n}}\right|=\frac{1}{q_{n}^{\tau_{n}\left(x_{0}\right)}} .
$$

The irrationality exponent (also called Diophantine approximation exponent or Diophantine order) of $x_{0}$ is

$$
\tau\left(x_{0}\right)=\limsup _{n \rightarrow+\infty} \tau_{n}\left(x_{0}\right) .
$$

If $x_{0}$ is irrational, then $\left|x_{0}-p_{n} / q_{n}\right|<1 / q_{n}^{2}$, so that $\tau_{n}\left(x_{0}\right)>2$, and $\tau\left(x_{0}\right) \geq 2$. Let us recall the following equivalent definition for the irrationality 
exponent of $x_{0}: \tau\left(x_{0}\right)$ is the supremum of the $\tau \in \mathbb{R}$ such that there exists infinitely many $(p, q) \in \mathbb{Z} \times \mathbb{N}^{*}$ such that $|x-p / q| \leq 1 / q^{\tau}$.

Theorem 1. If $x_{0} \in \mathbb{Q}$, then $h_{B}^{1}\left(x_{0}\right)=0$. Otherwise,

$$
h_{B}^{1}\left(x_{0}\right)=\frac{1}{\tau\left(x_{0}\right)} \text {. }
$$

Remark: Since almost every real number $x$ satisfies $\tau(x)=2$ (see e.g. Chap. 10.3 of [15]) it follows that $h_{B}^{1}$ takes the value $1 / 2$ almost everywhere. In the opposite direction, since quasi-every real number (in the sense of Baire categories) satisfies $\tau(x)=+\infty$, see [28], it follows that $h_{B}^{1}$ vanishes quasieverywhere (i.e. vanishes at least on a countable intersection of open dense subsets).

We now derive the consequence of Theorem 1 for multifractal analysis. Let $\operatorname{dim}(A)$ denote the Hausdorff dimension of the set $A$, with the convention $\operatorname{dim}(\emptyset)=-\infty$.

Definition 3. Let $p \in[1,+\infty)$ and $f \in L_{\text {loc }}^{p}(\mathbb{R})$. The level sets of $h_{f}^{p}$, denoted by $E_{H}^{p}$, are

$$
\forall H \in\left[-\frac{1}{p},+\infty\right], \quad E_{H}^{p}=\left\{x: \quad h_{f}^{p}(x)=H\right\} .
$$

The p-spectrum of $f$ is the function $\mathcal{D}_{f}^{p}:[-1 / p,+\infty] \rightarrow \mathbb{R} \cup\{-\infty\}$ defined by

$$
\mathcal{D}_{f}^{p}(H)=\operatorname{dim}\left(E_{H}^{p}\right) .
$$

In contradistinction with the Hölder case, few $p$-spectrums have been determined: Let us mention the characteristic functions of some fractal sets [21] and random wavelet series [2]; generic results (in the Baire and prevalence settings) for functions in a Sobolev space were obtained by A. Fraysse [16]; recently, 2-exponents of trigonometric series which are not locally bounded were obtained by S. Seuret and A. Ubis 33.

The precised formulation of Jarnik's theorem states that

$$
\operatorname{dim}\{x: \tau(x)=t\}=\frac{2}{t},
$$

see e.g. [18]. Therefore the 1-spectrum of $B$ will therefore follow from Theorem 1 .

Corollary 1. The 1-spectrum of $B$ is

$$
\mathcal{D}_{B}^{1}(H)= \begin{cases}2 H & \text { if } H \in[0,1 / 2], \\ -\infty & \text { else. }\end{cases}
$$

Remark: Since (5) also holds after restricting to the points $x$ inside a nonempty open interval, it follows that the multifractal spectrum of $B$ restricted to any interval $(a, b)$ of positive length is also given by (6). Following 
[4], B is an homogeneous multifractal function.

Theorem 11 is proved in Section 2. The computation of the 1-exponent is sharpened in Section 3 where the exact modulus of continuity of $B$ at badly approximable numbers is determined. Results concerning other notions of pointwise regularity are grouped in Section 4. Finally, we mention related open problems in Section 5 .

\section{Determination of the 1-exponent of $B$}

The fact that $B \in B M O$ implies a uniform lower bound on the 1-exponent. Indeed it follows from the John-Nirenberg inequality (or from Proposition 3 of [3]) that

$$
\exists C>0, \forall x, y: \quad|x-y| \leq \frac{1}{2}, \quad\left|\int_{x}^{y} B(t) d t\right| \leq C|x-y| \log \left(\frac{1}{|x-y|}\right)
$$

(here and in the following, the value of the constant $C$ may change from one line to the next). Thus, for $h<1 / 2$,

$$
\forall D, \quad \frac{1}{2 h} \int_{x_{0}-h}^{x_{0}+h}|B(x)-D| d x \leq C \log (1 / h)
$$

and finally,

$$
\forall x_{0}, \quad h_{B}^{1}\left(x_{0}\right) \geq 0
$$

Following [3, it will be convenient to define a function $\tilde{B}$ at rationals in the following way: If $x_{0} \in(0,1) \cap \mathbb{Q}$, then the continued fraction expansion (11) of $x_{0}$ stops at a rank $N$, and

$$
\tilde{B}\left(x_{0}\right)=\sum_{n=0}^{N-1}\left|p_{n-1}-q_{n-1} x_{0}\right| \log \left(\frac{p_{n-1}-x_{0} q_{n-1}}{q_{n} x_{0}-p_{n}}\right) ;
$$

for instance, for $N=1, \tilde{B}(1 / k)=\log k$.

The regularity of $B$ at rationals is a consequence of the following estimate of Balazard and Martin (Proposition 12 of [3]): Let $r=p / q$ with $p \wedge q=1$;

$$
\text { if }|h|<\frac{2}{3 q^{2}}, \frac{1}{h} \int_{r}^{r+h} B(x) d x=\frac{\log \left(e / q^{2}|h|\right)}{q}+\tilde{B}(r)+\mathcal{O}\left(q h \log \left(\frac{1}{q^{2}|h|}\right)\right)
$$

where the $\mathcal{O}$ is uniform (in $p, q$ and $h$ ). In particular, if $x_{0}=p / q$ is rational, then $\forall D$, for $h$ small enough,

$$
\int_{x_{0}-h}^{x_{0}+h}|B(x)-D| d x \geq \frac{h}{2 q} \log (1 / h),
$$

so that, at rationals $h_{B}^{1}\left(x_{0}\right)=0$. More precisely, by (77), for $C$ large enough, the function $C h \log (1 / h)$ is a uniform 1-modulus of continuity of $B$ (which will be defined further at Definition (4); and, up to the multiplicative constant, 
this is optimal, because it follows from (7) and (10) that $h \log (1 / h)$ is the order of magnitude of the left hand side of (10).

The regularity of $B$ at Cremer numbers (i.e. at irrationals that are not Brjuno numbers) follows from the fact that they are not Lebesgue points, see Proposition 14 of [3]. Thus, it follows from (4) that $h_{B}^{1}\left(x_{0}\right) \leq 0$ and, using (8), $h_{B}^{1}\left(x_{0}\right)=0$. Therefore, from now on, we can assume that $x_{0}$ is a Brjuno number, so that $B\left(x_{0}\right)$ is finite, and its values (pointwise and in the Lebesgue sense) coincide.

2.1. Global and pointwise irregularity of the Brjuno function. The idea for proving the irregularity of $B$ at Brjuno numbers is to reinterpret (91) as implying that some of its wavelet coefficients are large in the neighbourhood of the point considered, so that $B$ is irregular at those points. We will need a variant of the classical wavelet criterium (such as in [17]).

We assume in the following that $\psi$ is a bounded, compactly supported function satisfying

$$
\sup _{x \in \mathbb{R}}|\psi(x)| \leq 1 \quad \text { and } \quad \int_{\mathbb{R}} \psi(x) d x=0 ;
$$

such a function $\psi$ will be called an admissible wavelet. Let

$$
\forall a>0, \quad b \in \mathbb{R}, \quad \psi_{a, b}(x)=\psi\left(\frac{x-b}{a}\right) .
$$

If $f \in L_{l o c}^{1}(\mathbb{R})$, the continuous wavelet transform of $f$ is

$$
C_{f}(a, b)=\frac{1}{a} \int_{\mathbb{R}} f(x) \psi_{a, b}(x) d x .
$$

In order to obtain sharp results, we need to extend the notion of $T_{\alpha}^{p}$ regularity to general moduli of continuity. We start by defining the possible candidates: A function $\theta: \mathbb{R}^{+} \rightarrow \mathbb{R}^{+}$satisfies hypothesis $\mathcal{H}$ if

$(\mathcal{H}) \quad\left\{\begin{array}{l}\theta(0)=0, \\ \theta \text { is continuous and non-decreasing in a neighborhood of } 0 .\end{array}\right.$

Definition 4. Let $\theta$ be a function satisfying $\mathcal{H}$ and $f \in L_{l o c}^{p}(\mathbb{R}) ; \theta$ is a pmodulus of continuity of $f$ at $x_{0}$ if there exists a polynomial $P$ such that, for $\rho$ small enough,

$$
\left(\int_{x_{0}-\rho}^{x_{0}+\rho}\left|f(x)-P\left(x-x_{0}\right)\right|^{p} d x\right)^{1 / p} \leq \theta(\rho) .
$$

Note that $T_{\alpha}^{p}$ regularity corresponds to $\theta(\rho)=C \rho^{\alpha+1 / p}$.

Lemma 1. Let $\psi$ be an admissible wavelet, $p \in[1,+\infty]$ and $f \in L_{l o c}^{p}(\mathbb{R})$; let $\theta$ be a p-modulus of continuity of $f$ at $x_{0}$ satisfying

$$
\exists C>0, \forall \rho \in(0,1], \quad \theta(\rho) \geq C \rho^{1+1 / p} .
$$


Then

$$
\operatorname{supp}\left(\psi_{a, b}\right) \subset\left[x_{0}-\rho, x_{0}+\rho\right] \Longrightarrow\left|C_{f}(a, b)\right| \leq 2^{1-1 / p} \theta(\rho) \frac{\rho^{1-1 / p}}{a} .
$$

This result will be used for $p=1$ in order to prove the pointwise irregularity of the Brjuno function.

Proof. The growth condition on $\theta$ implies that we can restrict to polynomials $P$ of degree 0 . Since $\psi$ has a vanishing first moment,

$$
\forall D \in \mathbb{R}, \quad C_{f}(a, b)=\frac{1}{a} \int_{\mathbb{R}}(f(x)-D) \psi_{a, b}(x) d x .
$$

Using (11), we get

$$
\left|C_{f}(a, b)\right| \leq \frac{1}{a} \int_{x_{0}-\rho}^{x_{0}+\rho}|f(x)-D| d x \leq 2^{1-1 / p} \frac{\theta(\rho)}{a} \rho^{1-1 / p} .
$$

Applying (9) to $h$ and $h / 2$, we obtain that, if $0<h<2 / 3 q^{2}$, then

$$
\frac{1}{h} \int B(x) H\left(\frac{x-r}{h}\right) d x=\frac{\log 2}{q}+\mathcal{O}\left(q h \log \left(\frac{1}{q^{2} h}\right)\right),
$$

where $H=1_{[0,1 / 2]}-1_{[1 / 2,1]}$ is the Haar wavelet. Hence the following result holds.

Lemma 2. Let $r=p / q$ with $p \wedge q=1$. If $0<h<2 / 3 q^{2}$, then

$$
\left|C_{B}\left(h, \frac{p}{q}\right)-\frac{\log 2}{q}\right| \leq \tilde{C} q h \log \left(\frac{1}{q^{2} h}\right),
$$

where the wavelet used is the Haar wavelet and the constant $\tilde{C}$ is independent of $p, q$ and $h$.

We now introduce a notion of uniform irregularity associated with moduli of continuity for $p=1$.

Definition 5. Let $\theta$ be a function satisfying $\mathcal{H}$. A function $f \in L_{\text {loc }}^{1}(\mathbb{R})$ is uniformly $\theta$-irregular if

$$
\forall x_{0}, \forall P, \exists \rho_{n} \rightarrow 0: \quad \int_{x_{0}-\rho_{n}}^{x_{0}+\rho_{n}}\left|f(x)-P\left(x-x_{0}\right)\right| d x \geq \theta\left(\rho_{n}\right) .
$$

Proposition 1. There exists $A>0$ such that $B$ is uniformly $\theta$-irregular for $\theta(\rho)=A \rho^{3 / 2}$; and this result is optimal (i.e. $\theta(\rho)$ cannot be replaced by a $\left.o\left(\rho^{3 / 2}\right)\right)$.

The optimality of Proposition 1 will be proved in Section 3 by considering badly approximable numbers. 
Proof. Let $x_{0} \in \mathbb{R}$. First note that, if $x_{0} \in \mathbb{Q}$, then the result follows from (10). If $x_{0} \notin \mathbb{Q}$, we apply (14) to the sequence

$$
r_{n}=\frac{p_{n}}{q_{n}}
$$

of convergents of $x_{0}$. We now pick $h_{n}=\varepsilon / q_{n}^{2}$, where $\varepsilon$ is positive and such that $\tilde{C} \varepsilon \log (1 / \varepsilon) \leq 1 / 4$ (where $\tilde{C}$ is the constant in Lemma 2). It follows that

$$
C_{B}\left(h_{n}, r_{n}\right) \geq \frac{1}{4 q_{n}} .
$$

We now apply Lemma 1 with $a=h_{n}, b=r_{n}$ and $\rho_{n}=\left|x_{0}-r_{n}\right|+h_{n}$; if $\theta$ is a 1-modulus of continuity at $x_{0}$, then

$$
\left|C_{B}\left(h_{n}, r_{n}\right)\right| \leq \frac{\theta\left(\rho_{n}\right)}{h_{n}}
$$

which implies that

$$
\frac{1}{4 q_{n}} \leq \frac{\theta\left(\rho_{n}\right)}{h_{n}}
$$

Using that $\rho_{n} \leq 2 / q_{n}^{2}$ and $\theta$ is increasing, if follows that

$$
\frac{\varepsilon}{4 q_{n}^{3}} \leq \theta\left(\rho_{n}\right) \leq \theta\left(2 / q_{n}^{2}\right)=\frac{2 A \sqrt{2}}{q_{n}^{3}},
$$

hence a contradiction if $A$ is small enough.

Proposition 1 implies that the 1 -exponent satisfies $\forall x \in \mathbb{R}, h_{B}^{1}(x) \leq 1 / 2$; thus we can assume in the following that the polynomial in (12) boils down to a constant which has to be $B\left(x_{0}\right)$ as $x_{0}$ is a Brjuno number (recall that Brjuno numbers are Lebesgue points).

Let us now check that the same argument as in the proof of Proposition 1 yields an irregularity result at points $x_{0}$ for which $\tau\left(x_{0}\right)>2$. Recall that an irrational point $x_{0}$ is $\tau$-well approximable if

$$
\left|x_{0}-r_{n}\right| \leq \frac{1}{q_{n}^{\tau}}
$$

for infinitely many $n$ s.

Lemma 3. Let $\tau>2$. If $x_{0}$ is $\tau$-well approximable, then $\theta(\rho)=\frac{1}{8} \rho^{1+1 / \tau}$ is not a modulus of continuity of $B$ at $x_{0}$ (so that $\left.h_{B}^{1}\left(x_{0}\right) \leq 1 / \tau\right)$.

Proof. Assume that $\theta(\rho)=\frac{1}{8} \rho^{1+1 / \tau}$ is a modulus of continuity of $B$ at $x_{0}$. We pick $h_{n}=1 / q_{n}^{\tau}$. As above $C_{B}\left(1 / q_{n}^{\tau}, r_{n}\right) \sim \log (2) / q_{n}$ when $n \rightarrow+\infty$. We apply Lemma 1 with $a=h_{n}, b=r_{n}$ and $\rho_{n}=\left|x_{0}-r_{n}\right|+h_{n}$ so that $\rho_{n} \leq 2 / q_{n}^{\tau}$. We get $1 / 2 \leq 2^{1+1 / \tau} / 8$, hence a contradiction.

Recall that an irrational number $x_{0}$ is Diophantine if $\tau\left(x_{0}\right)<\infty$; Liouville numbers are the irrational numbers that are not Diophantine. It follows from 
Lemma 3 that the 1-exponent of the Brjuno function vanishes at Liouville numbers. Moreover if $x_{0}$ is such that $\tau\left(x_{0}\right)>2$, then Lemma 3 gives

$$
h_{B}^{1}\left(x_{0}\right) \leq \frac{1}{\tau\left(x_{0}\right)} \text {. }
$$

2.2. Pointwise regularity of the Brjuno function. We now prove regularity for $B$ at Diophantine numbers of $X=(0,1) \backslash \mathbb{Q}$.

We begin by recalling basic points about the continued fraction expansion of irrational numbers. First, the Gauss map $A: X \rightarrow X$ is defined by

$$
A(x)=\left\{\frac{1}{x}\right\}
$$

where $\{x\}=x-\lfloor x\rfloor$ denotes the fractional part of $x$ and $\lfloor x\rfloor$ its integer part of $x$. For $n \in \mathbb{N}$, we denote by $A^{n}$ the $n$-th iterate of $A$.

$$
\text { If } x \in X \text { and } n \geq 0, \quad A^{n}(x)=\frac{p_{n-1}(x)-x q_{n-1}(x)}{q_{n}(x) x-p_{n}(x)} \text { and }\left\lfloor A^{n}(x)\right\rfloor=a_{n}(x) \text {, }
$$

see e.g. [7] p. 40-41.

We will denote by $\mathfrak{c}\left[b_{1}, \ldots, b_{k}\right]$ the open sub-interval of $(0,1)$ with endpoints $\left[0 ; b_{1}, \ldots, b_{k}\right]$ and $\left[0 ; b_{1}, \ldots, b_{k-1}, b_{k}+1\right]$. These intervals are called cylinders of order $k$. Note that in a cylinder of order $k, A^{k}$ is continuous, and for all $j \leq k$ the functions $a_{j}, p_{j}$ and $q_{j}$ are constant. For $x \in X$, let

$$
\beta_{n}(x)=\left|x q_{n}(x)-p_{n}(x)\right|
$$

and

$$
\gamma_{n}(x)=\beta_{n-1}(x) \log \left(\frac{1}{A^{n}(x)}\right)
$$

so that

$$
B(x)=\sum_{n=0}^{\infty} \gamma_{n}(x)
$$

We have

$$
\beta_{n}(x)=\frac{1}{q_{k+1}(x)+A^{k+1}(x) q_{k}(x)},
$$

from which we get the well-known bounds (see e.g [7] p.42)

$$
\frac{1}{2 q_{n+1}(x)} \leq \beta_{n}(x) \leq \frac{1}{q_{n+1}(x)} \text {. }
$$

It follows that

$$
q_{n+1}(x) \leq q_{n}(x)^{\tau_{n}(x)-1} .
$$

Let us also recall (see Proposition 1 of [3]) that for $k \geq 1$,

$$
\frac{\log \left(q_{k+1}(x)\right)}{q_{k}(x)}-\frac{\log \left(2 q_{k}(x)\right)}{q_{k}(x)} \leq \gamma_{k}(x) \leq \frac{\log \left(q_{k+1}(x)\right)}{q_{k}(x)} .
$$


Let $x_{0} \in X$. In the sequel, $a_{k}, p_{k}, q_{k}$ denote the value at $x_{0}$ of the functions $a_{k}, p_{k}, q_{k}$. We need to estimate the integrals

$$
\int_{I} \gamma_{k}(t) d t
$$

where

$$
I=\left(x_{0}-\rho / 2 ; x_{0}+\rho / 2\right) \quad \text { with } \quad \rho>0 .
$$

These estimates will depend on an integer $K$ which is defined as follows: $K(=K(I))$ is the largest integer such that

$$
I \subseteq \mathfrak{c}\left[a_{1}, \ldots, a_{K}\right] .
$$

We also denote by $\left\{F_{k}\right\}_{k \geq 0}$ the sequence of Fibonacci numbers (i.e. $F_{0}=$ $\left.F_{1}=1, F_{n+2}=F_{n+1}+F_{n}\right)$.

Lemma 4. Let $x_{0} \in X$, let $\rho$ be such that $0<\rho<e^{-2}$ with $x_{0}-\rho / 2$ and $x_{0}+\rho / 2$ irrational. Let $I$ be the interval be given by (18) and $K$ the integer defined by (19). There exists an absolute constant $C>0$ such that for $K \geq 1$,

$$
\begin{aligned}
& \forall k<K, \quad \int_{I}\left|\gamma_{k}(x)-\gamma_{k}\left(x_{0}\right)\right| d x \leq C q_{k+1} \rho^{2}, \\
& \int_{I}\left|\gamma_{K}(x)-\gamma_{K}\left(x_{0}\right)\right| d x \leq C q_{K+1} \rho^{2} \log \left(q_{K+1}\right), \\
& \forall k>K, \quad \int_{I} \gamma_{k}(x) d x \leq C \frac{\rho}{F_{k-K}}\left(\frac{\log (1 / \rho)}{q_{K+1}}+\rho^{1 / 2}\right) .
\end{aligned}
$$

Proof. The bound (20) is exactly Proposition 7 of [3]. Propositions 9 and 10 of the same paper give bounds for the integrals $\int_{I} \gamma_{k}(x) d x$ for $k \geq K$, however (21) and (22) cannot be directly derived from them, but will be a consequence of their proofs. Let us recall the notations of [3]. The endpoints of the interval $\mathfrak{c}\left[a_{1}, \ldots, a_{K}\right]$ are

$$
\frac{p_{K}}{q_{K}} \text { and } \quad \frac{p_{K}+p_{K-1}}{q_{K}+q_{K-1}} .
$$

Up to some subset of $\mathbb{Q}, \mathfrak{c}\left[a_{1}, \ldots, a_{K}\right]$ is the union of the cylinders $\mathfrak{c}\left[a_{1}, \ldots, a_{K}, n\right]$ over $n \geq 1$. Any element $x$ of $\mathfrak{c}$ has a unique representation

$$
\left.x=\frac{s p_{K}+p_{K-1}}{s q_{K}+q_{K-1}} \quad \text { with } \quad s \in\right] 1 ;+\infty[.
$$

We set

$$
x_{0}+(-1)^{K} \rho / 2=\frac{u p_{K}+p_{K-1}}{u q_{K}+q_{K-1}} \text { and } x_{0}+(-1)^{K-1} \rho / 2=\frac{v p_{K}+p_{K-1}}{v q_{K}+q_{K-1}},
$$

and

$$
m=[u] \text { and } n=[v],
$$

so that $1 \leq m \leq a_{K+1} \leq n$. By maximality of $K$, we have $n>m$. Inequality (40) of [3] gives

$$
\rho \geq \frac{v-u}{6 q_{K}^{2} m n} .
$$


Let us now prove (21). We distinguish two cases.

First, suppose that $n \geq 2 m+1$. Then $v-u \geq(n-m) / 2$ and we obtain from (24) that

$$
\rho \geq \frac{1}{24 m q_{K}^{2}} \geq \frac{1}{24 a_{K+1} q_{K}^{2}} \geq \frac{1}{24 q_{K} q_{K+1}} .
$$

Proposition 9 of [3] and (17) give

$$
\int_{I} \gamma_{K}(x) \leq C \rho \frac{\log \left(q_{K+1}\right)}{q_{K}}
$$

From this we deduce

$$
\begin{aligned}
\int_{I}\left|\gamma_{K}(x)-\gamma_{K}\left(x_{0}\right)\right| d x & \leq \int_{I} \gamma_{K}(x) d x+\rho \gamma_{K}\left(x_{0}\right) \\
& \leq C \frac{\rho}{q_{K}} \log \left(q_{K+1}\right) \leq C \rho^{2} q_{K+1} \log \left(q_{K+1}\right)
\end{aligned}
$$

where the last inequality comes from (25).

Suppose now that $m \leq n \leq 2 m$. If $x \in I$, the derivative of $\gamma_{K}$ satisfies

$$
\gamma_{K}^{\prime}(x)=(-1)^{K-1}\left(q_{K-1}(x) \log \left(1 / A^{K}(x)\right)+\beta_{K}(x)^{-1}\right),
$$

so that

$$
\left|\gamma_{K}^{\prime}(x)\right| \leq C q_{K+1}(x)
$$

For $x \in I$, there exists $m \leq \ell \leq n$ such that $x \in \mathfrak{c}\left[a_{1}, \ldots, a_{K}, \ell\right]$ which yields

$$
q_{K+1}(x)=\ell q_{K}+q_{K-1} \leq n q_{K}+q_{K-1} \leq 2 a_{K+1} q_{K}+q_{K-1} \leq 2 q_{K+1} .
$$

By the mean-value theorem,

$$
\int_{I}\left|\gamma_{K}(x)-\gamma_{K}\left(x_{0}\right)\right| d x \leq C q_{K+1} \rho^{2}
$$

and the case $k=K$ is settled.

It remains to consider the case $k>K$. Let

$$
E=n-m+1
$$

If $E=2$, inequality (43) of [3] gives

$$
\int_{I} \gamma_{k}(x) d x \leq \frac{2 e}{q_{K+1} F_{k-K}} \rho \log (1 / \rho) .
$$

If $E \geq 3$, then $v-u \geq(n-m) / 2$ so that (24) gives $\rho \geq \frac{n-m}{12 q_{K}^{2} m n}$. Using

$$
\int_{I} \gamma_{k}(x) d x \leq 6 \frac{n-m}{q_{K}^{3} F_{k-K} m^{2} n}
$$

(see p. 213 of [3]), it follows that

$$
\int_{I} \gamma_{k}(x) d x \leq \frac{(12 \rho)^{3 / 2}}{F_{k-K}}\left(\frac{n}{(n-m) m}\right)^{1 / 2} \leq \frac{(12 \rho)^{3 / 2}}{F_{k-K}}
$$


We are now able to prove the following result.

Proposition 2. Let $x_{0}$ be a Diophantine number, and $\varepsilon>0$. There exists $C=C\left(x_{0}\right)>0$ and $\rho_{0}=\rho_{0}\left(x_{0}, \varepsilon\right)>0$ such that, if $0<\rho<\rho_{0}$, then

$$
\frac{1}{\rho} \int_{x_{0}-\rho / 2}^{x_{0}+\rho / 2}\left|B\left(x_{0}\right)-B(x)\right| d x \leq C \rho^{1 /\left(\tau\left(x_{0}\right)+\varepsilon\right)} \log (1 / \rho) .
$$

From (30) we deduce that, if $x_{0}$ is Diophantine, then for every $\varepsilon$ such that $0<\varepsilon<1 / 2, h_{B}^{1}\left(x_{0}\right) \geq 1 /\left(\tau\left(x_{0}\right)+\varepsilon\right)$, and consequently $h_{B}^{1}\left(x_{0}\right) \geq \frac{1}{\tau\left(x_{0}\right)}$ (as $\left.\tau\left(x_{0}\right) \geq 2\right)$ ) which ends the proof of Theorem 1. We now prove Proposition 2 .

Proof. As the set of irrational numbers is dense in $\mathbb{R}$, we may assume that $x_{0} \pm \rho / 2$ are both irrational. Let $\varepsilon>0$. There exists an integer $K_{0}=K_{0}\left(x_{0}, \varepsilon\right)$ such that

$$
\forall K \geq K_{0}, \quad \tau_{K}\left(x_{0}\right) \leq \tau\left(x_{0}\right)+\varepsilon .
$$

We will note $\tau_{k}\left(x_{0}\right)=\tau_{k}$ and $\tau\left(x_{0}\right)=\tau$. Following [3, $\delta_{k}=\delta_{k}\left(x_{0}\right)$ will denote the distance from $x_{0}$ to the endpoints of $\mathfrak{c}\left[a_{1}, \ldots, a_{k}\right]$, i.e.

$$
\delta_{k}=\min \left(\left|x_{0}-\frac{p_{k}}{q_{k}}\right|,\left|x_{0}-\frac{p_{k}+p_{k-1}}{q_{k}+q_{k-1}}\right|\right) .
$$

Proposition 4 of [3] gives

$$
\delta_{k} \leq \frac{1}{q_{k} q_{k+1}} \quad \text { and } \quad \delta_{k} \geq \begin{cases}\frac{1}{2 q_{k+1} q_{k+2}} & \text { if } a_{k+1}=1 \\ \frac{1}{2 q_{k} q_{k+1}} & \text { if } a_{k+1} \geq 2 .\end{cases}
$$

Let $K=K\left(x_{0}, \rho\right)$ be the largest integer such that $I=\left(x_{0}-\rho / 2 ; x_{0}+\rho / 2\right)$ is included in $\mathfrak{c}\left[a_{1}, \ldots, a_{K}\right]$. We have

$$
\frac{1}{2 q_{K+2} q_{K+3}} \leq \delta_{K+1}<\rho / 2
$$

so that $K \rightarrow+\infty$ when $\rho \rightarrow 0$. Let $0<\rho_{0}<e^{-2}$ be such that for all $0<\rho<\rho_{0}, K \geq \max \left(K_{0}, 1\right)$ and let us evaluate for $\rho<\rho_{0}$,

$$
\int_{I}\left|B\left(x_{0}\right)-B(x)\right| d x \leq \sum_{k \leq K} \int_{I}\left|\gamma_{k}\left(x_{0}\right)-\gamma_{k}(x)\right| d x+\rho \sum_{k>K} \gamma_{k}\left(x_{0}\right)+\sum_{k>K} \int_{I} \gamma_{k}(x) d x .
$$

Using (20) and (21), and since the sequence $\left\{q_{k}\right\}_{k \geq 0}$ grows (at least) exponentially, it follows that

$$
\begin{aligned}
\sum_{k \leq K} \int_{I}\left|\gamma_{k}\left(x_{0}\right)-\gamma_{k}(x)\right| d x & \leq C \rho^{2}\left(\sum_{k<K} q_{k+1}+q_{K+1} \log \left(q_{K+1}\right)\right) \\
& \leq C \rho^{2} q_{K+1} \log \left(q_{K+1}\right) .
\end{aligned}
$$


Now, using (22), we get

$$
\begin{aligned}
\sum_{k>K} \int_{I} \gamma_{k}(x) d x & \leq C \rho\left(\frac{\log (1 / \rho)}{q_{K+1}}+\rho^{1 / 2}\right) \sum_{k>K} \frac{1}{F_{k-K}} \\
& \leq C \rho\left(\frac{\log (1 / \rho)}{q_{K+1}}+\rho^{1 / 2}\right) .
\end{aligned}
$$

(because the sequence $\left\{F_{k}\right\}_{k \geq 0}$ of Fibonacci numbers grows exponentially).

Since $x_{0}$ is Diophantine, $\tau\left(x_{0}\right)<\infty$; therefore the sequence $\left(\tau_{k}\right)_{k \geq 0}$ is bounded. Using (16) and (17), we get for $k>K$,

$$
\left|\gamma_{k}\left(x_{0}\right)\right| \leq \frac{\log \left(q_{k+1}\right)}{q_{k}} \leq \frac{\log \left(q_{k}^{\tau_{k}-1}\right)}{q_{k}} \leq C \frac{\log \left(q_{k}\right)}{q_{k}},
$$

where $C$ depends on $x_{0}$. We deduce from this and again from the exponential growth of $\left\{q_{k}\right\}_{k \geq 0}$ that

$$
\sum_{k>K} \gamma_{k}\left(x_{0}\right) \leq C \frac{\log \left(q_{K+1}\right)}{q_{K+1}}
$$

Collecting these estimates we get

$$
\int_{I}\left|B\left(x_{0}\right)-B(x)\right| d x \leq C \rho\left(\rho q_{K+1} \log \left(q_{K+1}\right)+\frac{\log (1 / \rho)}{q_{K+1}}+\rho^{1 / 2}\right) .
$$

According to (16)

$$
q_{K+1} \leq q_{K}^{\tau_{K}-1}=\left|x-\frac{p_{K}}{q_{K}}\right|^{\left(1-\tau_{K}\right) / \tau_{K}} \leq \rho^{-1+1 / \tau_{K}},
$$

from which we deduce that $\log \left(q_{K+1}\right) \leq C \log (1 / \rho)$. If $a_{K+2} \geq 2$, according to (15) and (32),

$$
\frac{1}{q_{K+1}^{\tau_{K}+1}}=\left|x-\frac{p_{K+1}}{q_{K+1}}\right| \leq \frac{1}{q_{K+1} q_{K+2}} \leq 2 \delta_{K+1}<\rho,
$$

and if $a_{K+2}=1$ we get in the same way

$$
\frac{1}{q_{K+1}} \leq \frac{2}{q_{K+2}} \leq 2 \rho^{1 / \tau_{K+2}} .
$$

Inserting these estimates in (33), we finally get

$$
\int_{I}\left|B\left(x_{0}\right)-B(x)\right| d x \leq C \rho\left(\rho^{1 / \tau_{K}}+\rho^{1 / \tau_{K+1}}+\rho^{1 / \tau_{K+2}}\right) \log (1 / \rho),
$$

(note that, since $\tau_{K} \geq 2$, the term $\rho^{1 / 2}$ in (33) is not needed) and the conclusion follows from (31).

We now turn to the proof of Corollary 1. It follows from a precised version of Jarnik Theorem (which, initially, yields the Hausdorff dimensions of the sets of points with a given irrationality exponent). In order to state it, we need to recall the following notion of modified Hausdorff measure. 
Definition 6. Let $A \subset \mathbb{R}$. If $\varepsilon>0$ and $\delta \in[0,1]$, we denote

$$
M_{\varepsilon}^{\delta, \gamma}=\inf _{R}\left(\sum_{i}\left|A_{i}\right|^{\delta}\left|\log \left(\left|A_{i}\right|\right)\right|^{\gamma}\right)
$$

where $R$ is an $\varepsilon$-covering of $A$, i.e. a covering of $A$ by bounded sets $\left\{A_{i}\right\}_{i \in \mathbb{N}}$ of diameters $\left|A_{i}\right| \leq \varepsilon$ (the infimum is therefore taken on all $\varepsilon$-coverings). For any $\delta \in[0,1]$ and $\gamma \in \mathbb{R}$, the $(\delta, \gamma)$-dimensional outer Hausdorff measure of $A$ is

$$
m e s^{\delta, \gamma}(A)=\lim _{\varepsilon \rightarrow 0} M_{\varepsilon}^{\delta, \gamma} .
$$

Proposition 3. Let $a, b \in \mathbb{R}$ such that $a<b$ and

$$
E_{\tau}=\left\{x \in[a, b]:\left|x-\frac{p}{q}\right| \leq \frac{1}{q^{\tau}} \text { for infinitely many couples }(p, q)\right\} ;
$$

then

$$
\begin{array}{r}
\operatorname{dim}\left(E_{\tau}\right)=2 / \tau, \\
\operatorname{mes}^{2 / \tau, 2}\left(E_{\tau}\right)>0 .
\end{array}
$$

Note that the upper bound in (34) follows immediately using the natural covering by the intervals $\left[\frac{p}{q}-\frac{1}{q^{\tau}}, \frac{p}{q}+\frac{1}{q^{\tau}}\right]$, and (35) implies the lower bound, so that the only result that requires a proof is (35) $)$. A direct and elementary way in order to prove (35) is to follow step by step the proof of Theorems 10.3 and 10.4 of [15], and check that it actually yields not only a lower bound for the dimension of $E_{\tau}$, but the more precise result given by (35). A more conceptual proof consists in noticing that the sets $E_{\tau}$ are limsup sets, and that, by Dirichlet's theorem, $E_{2}=\mathbb{R}$; so that Proposition 3 actually is a particular case of the standard ubiquity techniques, see [6] and references therein, or Theorem 2 of [19].

Let us now check how Corollary 1 follows from Theorem 1 and Proposition 3. Let $a<b$ be given and let

$$
F_{t}=\{x \in[a, b]: \tau(x)=t\} .
$$

We need to prove that

$$
\forall t \in[2,+\infty], \quad \operatorname{dim}\left(F_{t}\right)=\frac{2}{t} .
$$

Clearly,

$$
F_{t}=\bigcap_{\tau<t} E_{\tau}-\bigcup_{\tau>t} E_{\tau} .
$$

It follows that $\forall \tau<t, F_{t} \subset E_{\tau}$, and Proposition 3 implies that $\operatorname{dim}\left(F_{t}\right) \leq 2 / t$. In order to obtain the lower bound, we will prove that $\mathrm{mes}^{2 / t, 2}\left(F_{t}\right)>0$. Indeed, $F_{t}$ contains the set

$$
G_{t}=E_{t}-\bigcup_{\tau>t} E_{\tau}
$$


since the sequence $E_{\tau}$ is decreasing, the union can be rewritten as a countable union of sets, which, by (34), all have a vanishing $m e s^{2 / t, 2}$ Hausdorff measure, so that $m e s^{2 / t, 2}\left(G_{t}\right)=\mathrm{mes}^{2 / t, 2}\left(E_{t}\right)>0$.

\section{BADLY APPROXIMABLE NUMBERS}

Theorem 1 can be interpreted as stating that the slower the sequence $q_{n}$ increases, the smoother $B$ is at $x_{0}$. We now prove that, indeed, the points for which the sequence $q_{n}$ grows as slowly as possible are the ones where $B$ is the smoothest.

An irrational number $x_{0}$ is badly approximable if the sequence of $\left\{a_{k}\right\}_{k \geq 0}$ is bounded, or, equivalently, if

$$
\exists C>0, \quad \forall p, q \neq 0, \quad\left|x_{0}-\frac{p}{q}\right| \geq \frac{C}{q^{2}} .
$$

It follows that $\tau\left(x_{0}\right)=2$; thus we already know that $h_{B}^{1}\left(x_{0}\right)=1 / 2$. We now sharpen this result. Recall that the definition of the modulus of continuity is given by (12).

Proposition 4. A point $x_{0} \in(0,1)$ is badly approximable if and only if there exists $C>0$ such that $\theta(\rho)=C \rho^{3 / 2}$ is a modulus of continuity of $B$ at $x_{0}$.

A consequence is the optimality of Proposition 1 (up to the multiplicative constant): Badly approximable numbers have the smallest possible modulus of continuity.

Proof. First note that a function which is a $o\left(\rho^{3 / 2}\right)$ cannot be a modulus of continuity at badly approximable numbers, as a consequence of Proposition 1. We now prove that, for $C$ large enough, $C \rho^{3 / 2}$ is a modulus of continuity at such a number. In this proof, the values of $C$ may change from one line to the next, but only depend on $x_{0}$. We will use the same notations $(I, K, E$, $\left.\delta_{K}\right)$ as in the proofs of Lemma 4 and Proposition 2. First note that

$$
\rho \leq\left|x_{0}-\frac{p_{K}}{q_{K}}\right| \leq \frac{1}{q_{K}^{2}}
$$

and also, as $x_{0}$ is badly approximable,

$$
\exists C: \quad \frac{\rho}{2}>\delta_{K+1} \geq \frac{1}{2 q_{K+2} q_{K+3}} \geq \frac{C}{q_{K}^{2}} .
$$

According to (20) and (37),

$$
\sum_{k<K} \int_{I}\left|\gamma_{k}(x)-\gamma_{k}\left(x_{0}\right)\right| d x \leq C \rho^{2} q_{K} \leq C \rho^{3 / 2}
$$


Let $k>K$. The proof of Proposition 10 of [3] p. 213 contains the following inequality for $E \geq 3$, which actually remains true for $E \geq 2$ :

$$
\int_{I} \gamma_{k}(t) d t \leq \frac{2}{q_{K}^{3} F_{k-K}} \sum_{m \leq \ell \leq n} \frac{1}{\ell^{3}}
$$

This inequality and (38) directly imply

$$
\sum_{k>K} \int_{I} \gamma_{k}(x) d x \leq C \rho^{3 / 2}
$$

As $1 / A^{k}\left(x_{0}\right)=a_{k+1}\left(x_{0}\right)+A^{k+1}\left(x_{0}\right)$, there exists $C>0$ such that for all $k \in \mathbb{N}, \log \left(1 / A^{k}\left(x_{0}\right)\right) \leq C$; using the exponential growth of the $\left(q_{k}\right)_{k \geq 0}$, we get

$$
\rho \sum_{k \geq K} \gamma_{k}\left(x_{0}\right) \leq C \rho \sum_{k \geq K} \frac{1}{q_{k}} \leq C \frac{\rho}{q_{K}} \leq C \rho^{3 / 2}
$$

To treat $\int_{I} \gamma_{K}(x) d x$, we use (39) of [3] :

$$
\int_{I} \gamma_{K}(x) d x \leq \frac{1}{q_{K}^{3}} \int_{A^{K}(I)} \log (1 / u) d u \leq \frac{1}{q_{K}^{3}} \int_{0}^{\left|A^{K}(I)\right|} \log (1 / u) d u \leq C \rho^{3 / 2},
$$

for $u \mapsto \log (1 / u)$ is decreasing on $(0,1]$ and $\int_{0}^{1} \log (1 / u) d u<\infty$. Collecting the estimates (39), (40), (41) and (42) we obtain that $C \rho^{3 / 2}$ is a modulus of continuity at $x_{0}$.

We now prove that badly approximable points are the only one for which the modulus of continuity is equivalent to $\rho^{3 / 2}$. Let $h_{n}=\left|x_{0}-p_{n} / q_{n}\right| ; x_{0}$ is not badly approximable if and only if there exists a subsequence $n(m)$ such that for $m \rightarrow \infty$,

$$
h_{n(m)}=o\left(\frac{1}{\left(q_{n(m)}\right)^{2}}\right) .
$$

The proof then follows the one of Proposition 1: On one hand, (14) implies that

$$
C_{B}\left(h_{n(m)}, \frac{p_{n(m)}}{q_{n(m)}}\right) \geq \frac{1}{4 q_{n(m)}} ;
$$

on other hand, applying Lemma 1 with $\rho_{n(m)}=2 h_{n(m)}$, we obtain that, if $C \rho^{3 / 2}$ is a modulus of continuity at $x_{0}$, then

$$
\frac{1}{4 q_{n(m)}} \leq \frac{C\left(\rho_{n(m)}\right)^{3 / 2}}{h_{n(m)}} \leq C 2^{3 / 2}\left(h_{n(m)}\right)^{1 / 2}
$$

which contradicts (43). 


\section{Additional pointwise Regularity Results}

We start by showing why the pointwise exponent used for positive measures is not relevant for $B$. Recall that, if $\mu$ is a positive Radon measure defined on $\mathbb{R}$, The local dimension of $\mu$ at $x_{0}$ is

$$
\underline{\operatorname{dim}}_{l o c}\left(\mu, x_{0}\right)=\liminf _{\rho \rightarrow 0^{+}} \frac{\log \mu\left(\left[x_{0}-\rho, x_{0}+\rho\right]\right)}{\log \rho} .
$$

The local dimension is well defined for the Brjuno function; however, it does not allow to capture possible changes in its pointwise regularity. Indeed, let us check that it is constant.

First, clearly, $\exists C>0$ such that $\forall x \in \mathbb{R}, B(x) \geq C$ because there is no cancellation in the series (2); so that $\forall x, \underline{\operatorname{dim}}_{l o c}(\mu, x) \leq 1$. On other hand, since $B \in B M O$, it follows immediately from (17) that $\forall x, \underline{\operatorname{dim}}_{l o c}(\mu, x) \geq 1$.

A drawback of using the local dimension is that, in contradistinction with the Hölder exponent, two measures $\mu$ and $\nu$ differing by a constant may have different exponents. Therefore this exponent often takes the value 1 , because the definition does not include (as in the Hölder case) the substraction of an appropriate polynomial. This explains why the $p$-exponent, which allows for this substraction, is better fitted to measure variations of regularity of $B$.

4.1. Hölder regularity of the primitive of $B$. The proof of Theorem 1 strongly uses (9), which estimates increments of the primitive of $B$; therefore a natural question is to wonder if it can yield its Hölder exponent.

Definition 7. Let $f: \mathbb{R} \rightarrow \mathbb{R}$ be a locally bounded function, $x_{0} \in \mathbb{R}$ and $\alpha \geq 0$. The function $f$ belongs to $C^{\alpha}\left(x_{0}\right)$ if there exist $C>0$ and a polynomial $P$ of degree less than $\alpha$ such that, for $\rho$ small enough,

$$
\sup _{\left|x-x_{0}\right| \leq \rho}\left|f(x)-P\left(x-x_{0}\right)\right| \leq C \rho^{\alpha} .
$$

The Hölder exponent of $f$ at $x_{0}$ is

$$
h_{f}\left(x_{0}\right)=\sup \left\{\alpha \geq 0 \mid f \in C^{\alpha}\left(x_{0}\right)\right\} .
$$

We denote by $\mathfrak{B}$ a primitive of $B$. A lower bound for $h_{\mathfrak{B}}$ is a consequence of the following classical result.

Lemma 5. Let $f \in L_{l o c}^{1}(\mathbb{R})$, and denote by $F$ a primitive of $f$. Then

$$
\forall x_{0} \in \mathbb{R}, \quad h_{F}\left(x_{0}\right) \geq h_{f}^{1}\left(x_{0}\right)+1 .
$$

Proof. We recall the proof for the sake of completeness. Suppose that $f \in$ $T_{\alpha}^{1}\left(x_{0}\right)$, let $P$ be the polynomial given by (3) , and denote by $Q$ the primitive of $P$ that vanishes at $x_{0}$. The primitive $F(x)=\int_{x_{0}}^{x} f(t) d t$ satisfies

$$
\begin{aligned}
|F(x)-Q(x)| & =\left|\int_{x_{0}}^{x}(f(t)-P(t)) d t\right| \\
& \leq \int_{x_{0}}^{x}|f(t)-P(t)| d t \leq C\left|x-x_{0}\right|^{\alpha+1}
\end{aligned}
$$


so that $F \in C^{\alpha+1}\left(x_{0}\right)$.

Note that, in general, equality does not hold in (45), as shown by the functions $|x|^{\alpha} \sin \left(|x|^{-\beta}\right)$ for $\alpha>-1$ and $\beta>0$; we will now check that equality holds everywhere in the case of the Brjuno function.

Proposition 5. If $x_{0} \in \mathbb{Q}$, then $h_{\mathfrak{B}}\left(x_{0}\right)=1$. Otherwise,

$$
h_{\mathfrak{B}}\left(x_{0}\right)=1+\frac{1}{\tau\left(x_{0}\right)} .
$$

In order to prove this result, we will need an irregularity criterium based on finite differences. We note

$$
\Delta_{2} f(x, h)=2 f\left(x+\frac{h}{2}\right)-f(x+h)-f(x) .
$$

Lemma 6. Let $f: \mathbb{R} \rightarrow \mathbb{R}$ be a continuous function; let $\alpha<2$ and $\gamma \geq 0$. Let $x_{0} \in \mathbb{R}$, and assume that there exist $\rho_{n}>0, h_{n}$, and $r_{n}$ such that

$$
\begin{gathered}
r_{n} \in\left[x_{0}-\rho_{n}, x_{0}+\rho_{n}\right], \quad \rho_{n} \rightarrow 0, \quad \text { and } \quad \frac{\rho_{n}}{\left|\log \rho_{n}\right| \gamma} \leq\left|h_{n}\right| \leq \rho_{n} . \\
\text { If }\left|\Delta_{2} f\left(r_{n}, h_{n}\right)\right| \geq\left|h_{n}\right|^{\alpha}, \quad \text { then } \quad h_{f}\left(x_{0}\right) \leq \alpha .
\end{gathered}
$$

Proof. Clearly, if $f$ is continuous, then the sup ess in (44) can be replaced by a sup. Therefore, if $f \in C^{\beta}\left(x_{0}\right)$ for a $\beta<2$, then there exists a polynomial $P$ of degree at most 1 and $r>0$ such that

$$
\forall x \in\left[x_{0}-r, x_{0}+r\right], \quad f(x)=P\left(x-x_{0}\right)+O\left(\left|x-x_{0}\right|^{\beta}\right) .
$$

Using (47) for $x=r_{n}, r_{n}+h_{n} / 2$ and $r_{n}+h_{n}$ in (46), we get

$$
\Delta_{2} f\left(r_{n}, h_{n}\right)=O\left(\rho_{n}^{\beta}\right)=O\left(\left|h_{n}\right|^{\beta}|\log | h_{n}||^{\beta \gamma}\right) .
$$

Therefore, if $\left|\Delta_{2} f\left(r_{n}, h_{n}\right)\right| \geq\left|h_{n}\right|^{\alpha}$, then $\forall \beta>\alpha, f \notin C^{\beta}\left(x_{0}\right)$.

Let us now prove Proposition 5. The case $x_{0} \in \mathbb{Q}$ follows from (9). If $x_{0} \notin \mathbb{Q}$, (45) and Theorem 1 imply that $h_{\mathfrak{B}}\left(x_{0}\right) \geq 1+1 / \tau\left(x_{0}\right)$. Note that (13) can be rewritten

$$
\text { If }|h|<\frac{2}{3 q^{2}} \text {, then } \frac{1}{h} \Delta_{2} \mathfrak{B}(r, h)=\frac{\log 2}{q}+\mathcal{O}\left(q h \log \left(\frac{1}{q^{2}|h|}\right)\right) .
$$

Let now $\tau \geq 2$ and assume that $x_{0}$ is $\tau$-well approximable. We can assume, by extracting a subsequence if necessary, that for all $n \geq 1,\left|x_{0}-p_{n} / q_{n}\right| \leq 1 / q_{n}^{\tau}$, and we pick for $r$ the sequence of convergents $r_{n}=p_{n} / q_{n}, \rho_{n}=1 / q_{n}^{\tau}$, and $h_{n}=1 / q_{n}^{\tau}\left(\log q_{n}\right)^{2}$. We obtain that

$$
\Delta_{2} \mathfrak{B}\left(r_{n}, h_{n}\right)=\frac{\log 2}{\tau^{2 / \tau}}\left(h_{n}\right)^{1+1 / \tau}\left|\log \left(h_{n}\right)\right|^{2 / \tau}(1+o(1)),
$$

and Lemma 6 implies that $h_{\mathfrak{B}}\left(x_{0}\right) \leq 1+1 / \tau$. 
4.2. The $p$-exponent of $B$ for $p>1$. Theorem 1 can be extended to $p$ exponents in the following way :

$$
\forall p \geq 1, \forall x_{0} \in \mathbb{R}, \quad h_{B}^{p}\left(x_{0}\right)=h_{B}\left(x_{0}\right) .
$$

We outline the proof of this result. Using the John-Nirenberg inequality,

$$
\exists C>0, \forall x, y: \quad|x-y| \leq \frac{1}{2}, \quad\left|\int_{x}^{y} B(t)^{p} d t\right|^{1 / p} \leq C|x-y|^{1 / p} \log \left(\frac{1}{|x-y|}\right),
$$

so that $\forall x_{0} \in(0,1), h_{B}^{p}\left(x_{0}\right) \geq 0$. On other hand, Hölder's inequality implies that

$$
h_{B}^{p}\left(x_{0}\right) \leq h_{B}^{1}\left(x_{0}\right)
$$

Hence it follows from Theorem 1 that if $x_{0}$ is a rational or a Liouville number, then $h_{B}^{p}\left(x_{0}\right)=0$.

Suppose now that $x_{0}$ is Diophantine. The results obtained in section 2.2 are based on the estimates (20), (26), (28) and (29) from [3]. They extend as follows: Let $\rho$ be such that $0<\rho<e^{-2}$ with $x_{0}-\rho / 2$ and $x_{0}+\rho / 2$ irrational, $I=\left(x_{0}-\rho / 2 ; x_{0}+\rho / 2\right)$, and $K, m, n, E$ the integers defined by (19), (223) and (27). There exists an absolute constant $C>0$ such that for $K \geq 1$,

$$
\begin{aligned}
& \forall k<K, \quad\left(\int_{I}\left|\gamma_{k}(x)-\gamma_{k}\left(x_{0}\right)\right|^{p} d x\right)^{1 / p} \leq C q_{k+1} \rho^{1+1 / p}, \\
&\left(\int_{I} \gamma_{K}(x)^{p} d x\right)^{1 / p} \leq C \frac{\rho^{1 / p} \log \left(q_{K+1}\right)}{q_{K}}, \\
& \forall k>K, \quad\left(\int_{I} \gamma_{k}(x)^{p} d x\right)^{1 / p} \leq C \frac{\rho^{1 / p} \log (1 / \rho)}{F_{k-K} q_{K+1}}, \text { if } E=2, \\
&\left(\int_{I} \gamma_{k}(x)^{p} d x\right)^{1 / p} \leq C \frac{(n-m)^{1 / p}}{F_{k-K} q_{K}^{1+2 / p} m^{1+1 / p} n^{1 / p}} \text { if } E \geq 3 .
\end{aligned}
$$

The proofs follow the same arguments as in the proofs of Propositions 7, 9, 10 of [3]. Doing so, easy extensions of Proposition 2 and Lemma 2 of the same paper will be requested. As the method is exactly the same to get these, we give them without proofs :

- Let $I \subseteq[0 ; 1]$ be an interval of length $h \leq e^{-p}$. We have

$$
\forall k \in \mathbb{N}, \quad \int_{I} \gamma_{k}(x)^{p} d x \leq e^{p} h \log ^{p}(1 / h) .
$$

- Let $m, n$ be integers such that $1 \leq m<n$. We have

$$
\sum_{m \leq \ell \leq n} \frac{1}{\ell^{p+2}} \leq 3 \frac{n-m}{m^{p+1} n}
$$

Starting from (50), (51), (52) and (53), one obtains the following extension of Lemma 4: Under the same hypothesis there exists an absolute constant 
$C>0$ such that for $p \geq 1, K \geq 1$,

$$
\begin{aligned}
& \forall k<K, \quad\left(\int_{I}\left|\gamma_{k}(x)-\gamma_{k}\left(x_{0}\right)\right|^{p} d x\right)^{1 / p} \leq C q_{k+1} \rho^{1+1 / p}, \\
&\left(\int_{I}\left|\gamma_{K}(x)-\gamma_{K}\left(x_{0}\right)\right|^{p} d x\right)^{1 / p} \leq C q_{K+1} \rho^{1+1 / p} \log \left(q_{K+1}\right), \\
& \forall k>K, \quad\left(\int_{I} \gamma_{k}(x)^{p} d x\right)^{1 / p} \leq C \frac{\rho^{1 / p}}{F_{k-K}}\left(\frac{\log (1 / \rho)}{q_{K+1}}+\rho^{1 / 2}\right) .
\end{aligned}
$$

The following extension of Proposition 2 follows : If $x_{0}$ is a Diophantine number and $\varepsilon>0$, there exists $C=C\left(x_{0}\right)>0$ and $\rho_{0}=\rho_{0}\left(x_{0}, \varepsilon\right)>0$ such that for $p \geq 1,0<\rho<\rho_{0}$,

$$
\left(\frac{1}{\rho} \int_{x_{0}-\rho / 2}^{x_{0}+\rho / 2}\left|B\left(x_{0}\right)-B(x)\right|^{p} d x\right)^{1 / p} \leq C \rho^{1 /\left(\tau\left(x_{0}\right)+\varepsilon\right)} \log (1 / \rho) .
$$

From (55) we infer the lower-bound $h_{B}^{p}\left(x_{0}\right) \geq 1 / \tau\left(x_{0}\right)$. Combined with (49) and Theorem 1, this yields $h_{B}^{p}\left(x_{0}\right)=1 / \tau\left(x_{0}\right)$.

\section{Concluding Remarks}

The present paper raises the problem of determining if Theorem 1 also applies for variants of the Brjuno function.

First, $B$ is one example of a family $B_{\alpha}$ introduced by J.-C. Yoccoz in [35], and further studied in [22, 23]: In the definition of $B$, the usual continued fraction algorithm is replaced by $\alpha$-continued fractions expansions, see [27]. A similar analysis as the one that we performed could be developed for $B_{\alpha}$. Note that uniform regularity results for differences of such functions have immediate consequences on their pointwise regularity; for example, $B_{1 / 2}-B \in$ $C^{1 / 2}$, cf. Theorem 4.6 of [23]; since the $p$-exponents of $B$ belong to $[0,1 / 2]$, it follows that $B_{1 / 2}$ shares the same $p$-exponent as $B$ (except perhaps for badly approximable points where Proposition 4 leaves room for a cancellation between moduli of continuity).

Other extensions are proposed in [22] where the logarithm in $B$ is replaced by another function. An important subcase consists of choosing $1 / x^{\beta}$ with $0<\beta<1$. In this case the corresponding Brjuno function does not belong to all $L^{p}$ spaces and its pointwise exponent can be studied for a restricted range of $p$ s only. Such a function can be seen as a fractional derivative of the corresponding Brjuno function (defined with a logarithm); we can therefore expect that (when defined) its $p$-exponent is $\frac{1}{\tau\left(x_{0}\right)}-\beta$; indeed, this would be true under the assumption that these Brjuno functions only display cusp singularities (i.e. if the pointwise regularity exponents of these functions are shifted by $\beta$ only after a fractional integration of order $\beta$, see [1]), a plausible assumption in view of Proposition 5 which asserts that it is the case for $B$ itself. 
The Brjuno function can be interpreted as the imaginary part of a complex analytic function $\mathcal{B}$, see Section 1.3 of [24]; a remarkable property of the real part of $\mathcal{B}$ is that it is a bounded function which is continuous except at rationals, where it has a left and a right limit. This property is shared with some Davenport series, which are of the form $\sum a_{n} \omega(n x)$, where $\omega(x)=\{x\}-1 / 2$ if $x \in \mathbb{R} \backslash \mathbb{Z}$ and $\omega(x)=0$ else. If $\left(a_{n}\right) \in l^{1}$, these series display jumps located at rational numbers, thus often leading to a pointwise regularity exponent related with Diophantine approximation, see [20] in which a multifractal analysis based on the Hölder exponent is developed, and where discontinuities at rationals play a key role. This indicates that a multifractal analysis may also be performed on $\operatorname{Re}(\mathcal{B})$ : Since, for $p \in(1, \infty)$, the Hilbert transform does not modify the value of the $p$-exponents, it follows that (48) (and hence Theorem 1) also holds for $\operatorname{Re}(\mathcal{B})$; thus all $p$-exponents of $\operatorname{Re}(\mathcal{B})$ coincide for $p>1$, except perhaps for $p=+\infty$. A natural conjecture therefore is that it is also the case for $p=+\infty$, i.e. that the Hölder exponent of $\operatorname{Re}(\mathcal{B})$ is

$$
\begin{cases}h_{\operatorname{Re}(\mathcal{B})}\left(x_{0}\right)=0 & \text { for } x_{0} \in \mathbb{Q} \\ h_{\operatorname{Re}(\mathcal{B})}\left(x_{0}\right)=\frac{1}{\tau\left(x_{0}\right)} & \text { otherwise }\end{cases}
$$

The result clearly holds for $x_{0} \in \mathbb{Q}$, because $\operatorname{Re}(\mathcal{B})$ is discontinuous at rational points. Additionally, since any function satisfies $h_{f}\left(x_{0}\right) \leq h_{f}^{p}\left(x_{0}\right)$, it follows that if $x_{0} \notin \mathbb{Q}$, then $h_{R e(\mathcal{B})}\left(x_{0}\right) \leq 1 / \tau\left(x_{0}\right)$.

\section{REFERENCES}

[1] Abry, P., Jaffard, S., Leonarduzzi, R., Melot, C., and Wendt, H.: New exponents for pointwise singularity classification. In Proc. Fractals and Related Fields III, S. Seuret and J. Barral, Eds., to appear (2017)

[2] Abry, P., Jaffard, S., Leonarduzzi, R., Melot, C., and Wendt, H.: Multifractal analysis based on $p$-exponents and lacunarity exponents. In: Fractal geometry and stochastics 70, Progr. Probab. Birkhäuser/Springer, 279-313 (2015)

[3] Balazard, M., and Martin, B.: Comportement local moyen de la fonction de Brjuno. Fund. Math. 218, 193-224 (2012)

[4] Barral, J., Durand, A., Jaffard, S. and Seuret, S.: Local multifractal analysis. Fractal Geometry and Dynamical Systems in Pure and Applied Mathematics II: Fractals in Applied Mathematics D. Carfi, M.L. Lapidus, E.P.J. Pearse, and M. van Frankenhuijsen, Eds., Contemp. Math., AMS, 601, 31-64 (2013)

[5] Barral, J., Berestycki, J., Bertoin, J., Fan, A. H., Haas, B., Jaffard, S., Miermont, G., and Peyrière, J.: Quelques interactions entre analyse, probabilités et fractals. Panoramas et Synthèses 32, Société Mathématique de France, Paris (2010)

[6] Beresnevich, V., Dickinson, D., and Velani, S., Measure theoretic laws for limsup sets, Mem. Amer. Math. Soc. 179 (846), 1-91 (2006)

[7] Billingsley, P.: Ergodic theory and information. John Wiley \& Sons, Inc., New YorkLondon-Sydney, 1965.

[8] Brjuno, A. D.: Analytic form of differential equations. I, II. Trudy Moskov. Mat. Obšč. 25, 119-262 (1971); ibid. 26, 199-239 (1972) 
[9] Buff, X., and Chéritat, A.: The Brjuno function continuously estimates the size of quadratic Siegel disks. Ann. of Math. 164, 265-312 (2006)

[10] Calderón, A.-P., and Zygmund, A.: Local properties of solutions of elliptic partial differential equations. Studia Math. 20, 171-225 (1961)

[11] Chamizo, F.: Automorphic forms and differentiability properties. Trans. Amer. Math. Soc. 356, 1909-1935 (2004)

[12] Chamizo, F., Petrykiewicz, I. and Ruiz-Cabello, I.: The Hölder exponent of some Fourier series. J. Fourier Anal. Appl. 1-20 (2016)

[13] Chamizo, F., and Ubis, A.: Multifractal behavior of polynomial Fourier series. Adv. Math. 250, 1-3 (2014)

[14] Cheraghi, D., and Chéritat, A.: A proof of the Marmi-Moussa-Yoccoz conjecture for rotation numbers of high type. Invent. Math. 202 (2), 677-742 (2015)

[15] K. Falconer, Fractal geometry: Mathematical foundations and applications John Wiley \& Sons Inc., New York, 2nd edition (2003)

[16] Fraysse, A.: Generic validity of the multifractal formalism. SIAM J. Math. Anal. 39 (2), 593-607 (2007)

[17] Jaffard, S.: Exposants de Hölder en des points donnés et coefficients d'ondelettes. C. R. Acad. Sci. Paris Sér. I Math. 308 (4), 79-81 (1989)

[18] Jaffard, S.: The spectrum of singularities of Riemann's function. Rev. Mat. Iberoamericana 12 (2), 441-460 (1996)

[19] Jaffard, S: The multifractal nature of Lévy processes. Probab. Theory Relat. Fields 114, 207-227 (1999).

[20] Jaffard, S.: On Davenport expansions. In Fractal geometry and applications: a jubilee of Benoît Mandelbrot. Part 1, 72 Proc. Sympos. Pure Math. Amer. Math. Soc., Providence, RI, 273-303 (2004)

[21] Jaffard, S., and Mélot, C.: Wavelet analysis of fractal boundaries. Comm. Math. Phys. 258 (3), 513-565 (2005)

[22] Luzzi, L., Marmi, S., Nakada, H., and Natsui, R.: Generalized Brjuno functions associated to $\alpha$-continued fractions. J. Approx. Theory 162 (1), 24-41 (2010)

[23] Marmi, S., Moussa, P., and Yoccoz, J.-C.: The Brjuno functions and their regularity properties. Comm. Math. Phys. 186 (2), 265-293 (1997)

[24] Marmi, S., Moussa, P., and Yoccoz, J.-C.: Complex Brjuno functions. J. Amer. Math. Soc. 14 (4), 783-841 (2001)

[25] Marmi, S., Moussa, P., and Yoccoz, J.-C.: Some Properties of Real and Complex Brjuno Functions. Frontiers in Number Theory, Physics and Geometry I: On Random Matrices, Zeta Functions and Dynamical Systems, P. Cartier, B. Julia, P. Moussa and P. Vanhove Eds., Springer-Verlag: 603-628 (2006)

[26] Meyer, Y.: Wavelets, vibrations and scalings. 9 CRM Monograph Series. American Mathematical Society, Providence, RI (1998)

[27] Nakada, H.: Metrical theory for a class of continued fraction transformations and their natural extensions. Tokyo J. Math., 4 (2), 399-426 (1981)

[28] J. Oxtoby, Measure and category, Springer, Grad. Texts Math. 2 (1971)

[29] Pesin, Y.: Dimension theory in dynamical systems. Chicago Lectures in Mathematics. University of Chicago Press, Chicago, IL, Contemporary views and applications, (1997)

[30] Petrykiewicz, I.: Hölder regularity of arithmetic fourier series arising from modular forms. arXiv:1311.0655.

[31] Petrykiewicz, I.: Differentiability of arithmetic Fourier series arising from Eisenstein series. Ramanujan J. 42 (3), 527-581 (2017)

[32] Rivoal, T., and Roques, J.: Convergence and modular type properties of a twisted Riemann series. Unif. Distrib. Theory 8 (1), 97-119 (2013) 
[33] Seuret, S., and Ubis, A.: Local $L^{2}$-regularity of Riemann's Fourier series. to appear in Ann. Inst. Fourier.

[34] Siegel, C. L.: Iteration of analytic functions. Ann. of Math. 43 (2), 607-612 (1942)

[35] Yoccoz, J.-C.: Théorème de Siegel, nombres de Bruno et polynômes quadratiques. Astérisque 231 Petits diviseurs en dimension 1 3-88 (1995) 